\title{
Diffraction and Vector Mesons: Summary
}

\author{
Uta Klein $^{1}$ and Leszek Motyka ${ }^{2,3}$ \\ 1- University of Liverpool, Oliver Lodge Laboratory, L69 7ZE, England, U.K. \\ 2- II. Institute for Theoretical Physics, Hamburg University, \\ Luruper Chaussee 149, 22761 Hamburg, Germany \\ 3- Institute of Physics, Jagellonian University, \\ Reymonta 4, 30-059 Kraków, Poland
} We summarize recent experimental results and theoretical developments related to the
topics of diffraction and vector mesons discussed at the DIS2007 Workshop.

\section{Preface}

For nearly two decades, diffractive phenomena studied at the high energy frontier in $p \bar{p}$ collisions at the Tevatron and in ep collisions at HERA are shaping and challenging our understanding of the underlying dynamical quantum processes. Experimentally, diffractive processes are characterized by large 'rapidity gaps', regions of (pseudo-)rapidity in which no hadrons are produced, and/or by a beam particle $(p / \bar{p})$ which remains intact after the scatter. Large gaps in the final state may occur by the exchange of a color singlet object with vacuum quantum numbers, historically referred to as the Pomeron. The spectacular phenomenon of hard diffraction provides a unique way to probe QCD dynamics at low- $x$ and to interpret diffractive reactions by the exchange of partons, mainly gluons, of the interacting nucleon. A particularly exciting feature of diffractive Deep Inelastic Scattering (dDIS) is its relation to unitarity effects in hard QCD scattering amplitudes such as are implemented in modern QCD inspired saturation models and within the $k_{T}$ factorization framework in perturbative QCD (pQCD). An important merit of the $k_{T}$ factorization approach and the saturation model is a unified picture of inclusive and diffractive DIS that they provide. Complementary, in the collinear framework of pQCD, the Operator Product Expansion, inclusive hard diffraction is encoded in diffractive parton densities evolving according to the DGLAP evolution equations. Both frameworks are successfully used to describe data, however, the collinear approach doesn't connect inclusive and diffractive cross sections provided by the $S$-matrix unitarity.

Exclusive diffractive processes are excellent tools to probe the proton structure beyond the reach of inclusive DIS. Exclusive production of mesons, vector mesons and real photons in $e p$ scattering contains combined information about longitudinal momentum distributions of partons and their position on the transverse plane as encoded in Generalized Parton Distribution functions (GPDs). In the high energy and/or very small Bjorken- $x$ regime, exclusive processes can be intimately linked to inclusive DIS in the light-cone color dipole picture. This regime is dominated by the exchange of a hard pQCD Pomeron while data at lower energies are strongly affected by non-vacuum exchanges. A wealth of HERA data established the gross features of the pQCD based description of diffractive vector meson production as for example $\left(Q^{2}+M^{2}\right)$ as the hard scale for small-t diffraction. The s-channel helicity non-conservation in high energy small- $t$ diffractive scattering may be regarded as one of the important observations at HERA. 
Recently, efforts are made to understand exclusive processes in the collinear factorization beyond leading order. Systematic studies of NLO corrections clearly show that the perturbative expansion is stable at low and moderate collision energies but at large energies (that is at small Bjorken- $x$ ) the NLO are enhanced by $\log (1 / x)$ and become large. This calls for further developments of the NLO $k_{T}$-factorization formalism at small $x$ as performed in the Balitsky-Fadin-Kuraev-Lipatov (BFKL) frame.

Diffractive reactions constitute a significant fraction of the hadronic and electroproduction cross sections. With increasing statistics, improved instrumentation and better detector understanding, many important observations could be made and further established, e.g. regarding the diffractive structure function of the Pomeron and the breakdown of QCD factorization in hard diffraction between $e p$ at HERA and $p \bar{p}$ at Tevatron. The applicability of the hard diffractive factorization to semi-inclusive processes, e.g. to the diffractive dijet or diffractive charm photoproduction is not proven and recent, more precise HERA data are challenging the validity of factorization in hard diffractive dijet photoproduction.

The information extracted from diffraction in $e p$ collisions, mainly the dPDFs, may be used to calculate the hard diffractive subprocess in $p p$ and $p \bar{p}$ scattering. Due to nonperturbative effects, like proton remnant rescattering, diffractive factorization does not hold in hadronic collisions and the pattern of factorization breaking could be probed. In particular it is interesting to test the hypothesis of universal soft proton remnant rescattering that may be factorized out from the hard diffractive amplitude. Recent, precise HERA data on leading neutron production in DIS and in photoproduction give further insight into rescattering models which are necessary to understand gap-survival probabilities in high energy diffractive hadronic collisions. The hard hadronic diffraction is interesting not only because it is sensitive to the dynamics of QCD at large parton densities but also because new physics may be probed in a unique way in diffractive scattering at the LHC. The most important example is central exclusive diffractive Higgs boson production, a measurement that may provide a clean signal and facilitate a precise determination of the Higgs boson quantum numbers, its mass and width. In order to perform such measurements, vivid activities are ongoing on designing new forward and very forward detectors at the LHC.

In the following, we report on selected topics on latest experimental and theoretical developments as were presented to the session 'Diffraction and Vector Mesons' of this Workshop. The slides and more plots can be found in [1].

\section{$2 \quad$ Hard diffraction in $e p$ scattering}

At HERA, individual proton constituents may be resolved with a hard (that is small) probe provided by a virtual photon mediating $e^{ \pm} p$ scattering. Virtual photons carrying large negative momentum transfer, say $Q^{2}=-q^{2}=10 \mathrm{GeV}^{2}$, to a single parton are expected to destroy the proton with a probability very close to unity while final-state particles are uniformly emitted between the struck quark and the proton remnant. However, it is now a well-established fact that the proton emerges intact in a sizable fraction of DIS events: between $30 \%$ and $5 \%$ when the photon virtuality increases from $Q^{2} \simeq 0$ to $Q^{2} \simeq 190$ $\mathrm{GeV}^{2}$. The signature of hard diffraction at HERA is depicted in Fig. 1, characterized by a large separation in rapidity between the proton remnant $\left(p^{\prime}\right)$ and any other hadronic activity $(X)$ in the event. Diffractive scattering shows up as a peak at large fractions of the outgoing to the incoming proton momentum, $x_{L} \sim 1$, and small fractions of the proton momentum carried by the Pomeron, $x_{\mathbb{P}}=1-x_{L} \lesssim 0.01$. Diffractive production 
in the $t$ channel, where $t=\left(p-p^{\prime}\right)^{2}$ is the four-momentum transfer at the proton vertex, leads to an approximately constant $\ln M_{X}^{2}$ distribution for the particles of the system $X$ observed in the detector, in strong contrast to the exponential fall-off as seen for non-peripheral DIS events. The fraction of the Pomeron momentum carried by the struck quark, $\beta$, is related to the Bjorken- $x, x_{\mathrm{Bj}}$, via $\beta=x_{\mathrm{Bj}} / x_{\mathbb{P}}$. The fractional energy transferred to the proton in its rest system is $y$, the c.m. energy of the $\gamma^{*} p$ system is given with $W$.

The described signatures are used by both $\mathrm{H} 1$ and ZEUS collaborations for the measurements of hard diffraction in $e p$ collisons: either via event selections based on the detection of the outgoing proton with forward (leading) proton spectrometers (H1 FPS, ZEUS LPS methods) and on the detection of a large rapidity gap (LRG method) or via the determination of the mass

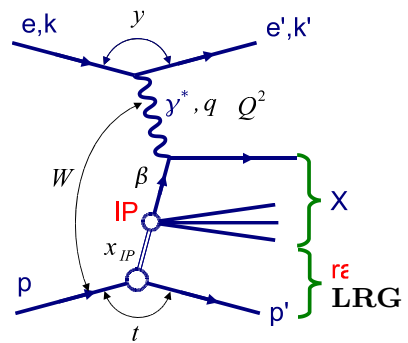

Figure 1: Schematic illustration of the diffractive contribution in neutral current DIS; plot from [2]. distribution $\ln M_{X}^{2}$ of the diffractively produced hadronic system where background and non-diffractive DIS contributions are subtracted statistically ( $M_{X}$ method).

At the workshop latest results of the HERA collaborations H1 and ZEUS were lively discussed, now with emphasis on results based on finalized HERA I data statistics. Using such larger data samples, a more detailed exploration of hard diffractive phenomena at HERA beyond the scope of the 'flagship' inclusive channel could be done. Those are studies of diffractive dijets in DIS and of diffractive dijets and open charm in photoproduction.

ZEUS reported [2] new results on the inclusive diffractive dissociation of virtual photons in events with a large rapidity gap and in events with a measured leading proton using integrated luminosities of $45.4 \mathrm{pb}^{-1}$ and of $32.6 \mathrm{pb}^{-1}$, respectively. The LRG (LPS) data cover photon virtualities $2<Q^{2}<305(120) \mathrm{GeV}^{2}$ and $2<M_{X}<25$ (40) GeV. LRG events were selected with $x_{\mathbb{P}}<0.02$, LPS events were required to have a proton detected in the leading proton spectrometer carrying at least $90 \%$ of the incoming proton energy. The $t$ integrated, reduced cross sections $\sigma_{r}^{D(3)}$ show good agreement between the results extracted with the LPS method and the LRG method. The agreement between ZEUS LPS and recently published H1 FPS results is fair keeping in mind a $10 \%$ normalization uncertainty of each of the data sets. There is also good agreement between both ZEUS LRG and recently published H1 LRG $\sigma_{r}^{D(3)}$ data after correcting for the different $p$ dissociation contribution per experiment; here the ZEUS data have been normalized to the H1 data. The ratio of the diffractive structure functions $F_{2}^{D(3)}$ from LPS/FPS and LRG is about $80 \%$. It thus allows an experimental estimate of the $p$ dissociation contribution with a precision limited by the $10 \%$ normalization uncertainties of the LPS/FPS methods.

Using the proton tag methods the exponential $t$ dependence of the cross section could be measured with a $b$ slope of $7 \pm 0.3 \mathrm{GeV}^{-2}$ (ZEUS) without any observed dependence on $Q^{2}$, $M_{X}$ and $x_{\mathbb{P}}$ in the measured region, $x_{\mathbb{P}}<0.01$. Within Regge phenomenology the exchange can be described by an effective Pomeron trajectory. From the $x_{\mathbb{P}}$ dependence of the diffractive structure function $F_{2}^{D(4)}$ and $F_{2}^{D(3)}$ a Pomeron intercept of $1.1 \pm 0.04$ in good agreement with $\mathrm{H} 1$ was measured. The Pomeron slope was found to be consistent with zero within a total uncertainty of about $0.1 \mathrm{GeV}^{-2}$ but significantly smaller than the value of $0.25 \mathrm{GeV}^{-2}$ 
found in soft hadronic interactions. The LPS data could be well described by a Regge fit in terms of Pomeron and Reggeon contributions with $x_{\mathbb{P}}$ and $t$ dependent flux factors and and $\beta$ and $Q^{2}$ dependent structure functions.

Furthermore ZEUS reported [3] new studies on inclusive and diffractive DIS based on the $M_{X}$ method for masses $1.2<M_{X}<$ $30 \mathrm{GeV}$ in an extended kinematic range of $25<Q^{2}<35 \mathrm{GeV}^{2}$ and of $45<Q^{2}<220 \mathrm{GeV}^{2}$ with an integrated luminosity of $11 \mathrm{pb}^{-1}$ and $52.4 \mathrm{pb}^{-1}$, respectively. Using a Forward Plug Calorimeter at HERA I, ZEUS diffractive analyzes profit from the measurement of the energy of particles in the pseudorapidity range $\eta \approx 4.0-5.0$ limiting the mass of the diffractively produced nucleonic system to $M_{N} \leq 2.3 \mathrm{GeV}$; H1 quotes $M_{N}<1.6 \mathrm{GeV}$ for the protondissociation system. A comparison for two values of proton momentum fractions of the Pomeron between the $\left(Q^{2}, \beta\right)$ dependence of $x_{\mathbb{P}} F_{2}^{D(3)}$ obtained with the LRG method (H1) and the $M_{X}$ method is shown in Fig. 2. The diffractive structure function values extracted with the different methods and different detectors show fair agreement for the $Q^{2}$ dependence except for a few bins. Observed differences at higher $x_{\mathbb{P}}>$ 0.01 (not shown here) may be affected by Reggeon contributions which could enter differently in the $M_{X}$ and LRG methods. In any case, the comparison and the consistency of these measurements is an important step forward in our understanding of an overall picture of hard diffraction at HERA. A next step could be a
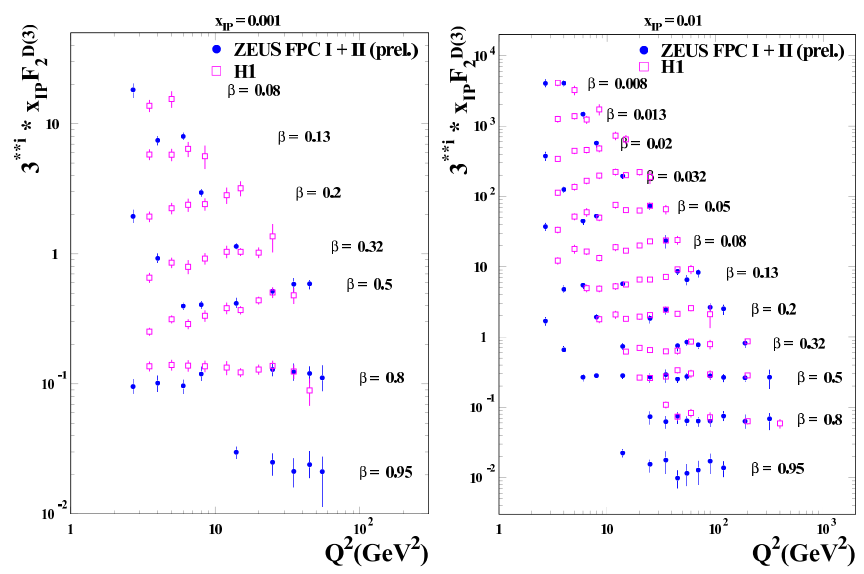

Figure 2: Comparison of the $\left(Q^{2}, \beta\right)$ dependence of $x_{\mathbb{P}} F_{2}^{D(3)}$ obtained with the LRG method (H1) and the $M_{X}$ method (ZEUS, shifted to centers of $\mathrm{H} 1 \mathrm{bins}$ ) for $x_{\mathbb{P}}=0.001$ (left) and $x_{\mathbb{P}}=0.01$ (right); plot from [3].

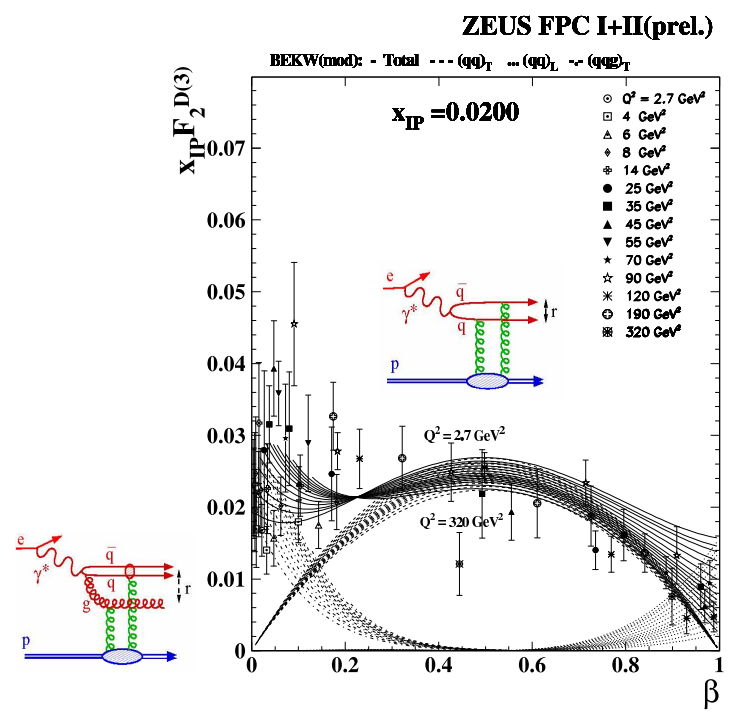

Figure 3: Compilation of the $\beta$ dependence of $x_{\mathbb{P}} F_{2}^{D(3)}$ for various $Q^{2}$ values but fixed $x_{\mathbb{P}}=0.02$. The curves are resulting from a BEKW fit (full) including the contributions from $q \bar{q}$ (depicted with an insert) for transverse (dashed) and longitudinal (dotted) photons as well as from $q \bar{q} g$ (depicted with an insert) for transverse photons (dashed-dotted); plot from [3]. 
comparison within a QCD NLO fit although this requires the assumption that the diffractive structure function factorizes in a term depending only on $x_{\mathbb{P}}$ and another term depending only on $\beta$ and $Q^{2}$. The LRG method results are consistent with this assumption but the $M_{X}$ method results indicate a breaking of the Regge (or proton vertex) factorization.

A good description of the $M_{X}$ method values of $x_{\mathbb{P}} F_{2}^{D(3)}$ could be obtained with a 5 parameter fit according to a modified BEKW model [3], see Fig. 3, which gives an intuitive picture in terms of contributions from the fluctuations of the incoming photon into dipoles: transverse photons to $q \bar{q}$, longitudinal photons to $q \bar{q}$, and transverse photons to $q \bar{q} g$. The dipole interacts with the target proton via two-gluon exchange. As expected from the model, the $q \bar{q}$ contributions from transverse photons dominate for $0.2<\beta<0.9$ while contributions from $q \bar{q} g$ dipoles become important at smaller $\beta \leq 0.15$. Only in the region close to $\beta$ near 1 contributions from longitudinal photons may become important.
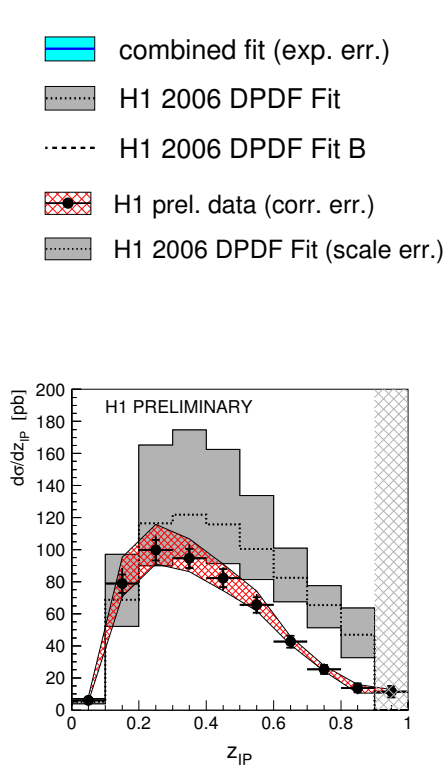
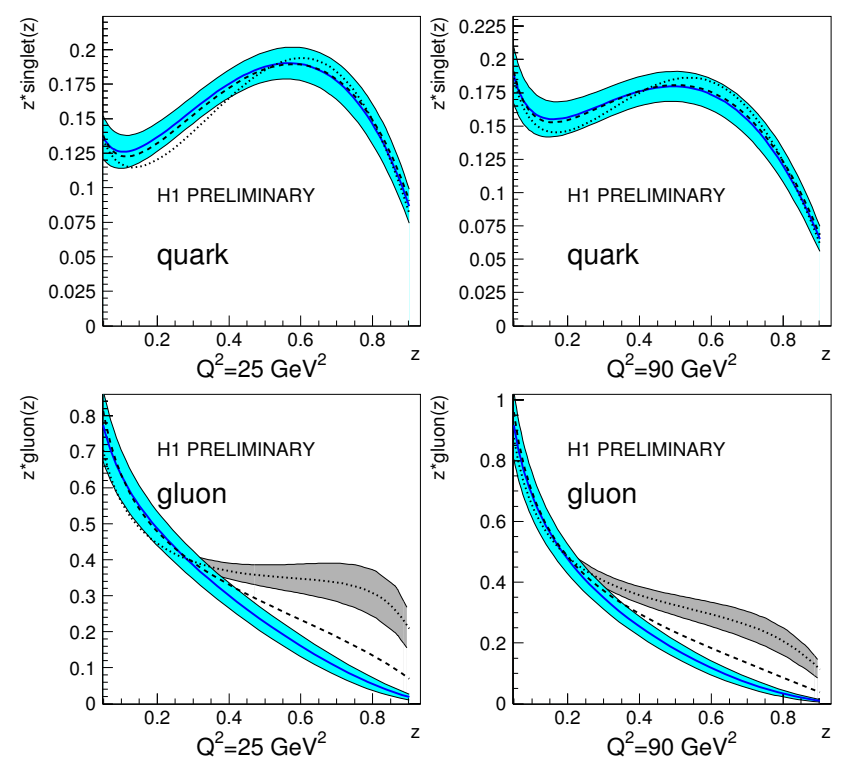

Figure 4: Left: Cross section $d \sigma / d z_{\mathbb{P}}$ of diffractive dijets in DIS with hatched band of correlated systematic uncertainties compared to NLO QCD predictions based on H1 2006 dPDFs with grey band indicating the scale uncertainty. Right: NLO QCD fit results for diffractive singlet (top) and gluon (bottom) distributions for two $Q^{2}$ scales, plots from [4].

At the workshop preliminary dijet cross sections in diffractive DIS were presented by H1 [4] selecting jets with transverse momenta, $p_{T_{1}}>5.5 \mathrm{GeV}$ and $p_{T_{2}}>4.0 \mathrm{GeV}$. The data span a broad kinematic range of $4<Q^{2}<80 \mathrm{GeV}^{2}, 0.1<y<0.7$ and $x_{\mathbb{P}}<0.03$. The data are in good agreement with a similar analysis performed by ZEUS ZEUS [5]. The $p_{T}$ and $x_{\mathbb{P}}$ dependencies are well described by the recently published H1 NLO QCD fits 2006 $\mathrm{A}$ and B. However, the $z_{\mathbb{P}}\left(z_{\mathbb{P}}=\beta\right)$ dependence, see Fig. 4 left, allows to further constrain the diffractive gluon distribution at $\beta>0.4$, a region where inclusive diffractive data have little sensitivity and the systematic uncertainties are high. A simultaneous NLO QCD fit of diffractive inclusive and dijet DIS cross sections result in a very good description of both data sets thus supporting QCD factorization. The extracted dPDFs are shown in Fig. 4 right. 
The diffractive singlet distribution is basically unchanged. However the diffractive gluon distribution of the combined fit is closer to the H1 2006 fit B but with reduced experimental uncertainty at large $z_{\mathbb{P}}$ due to the inclusion of the diffractive dijet data in DIS.
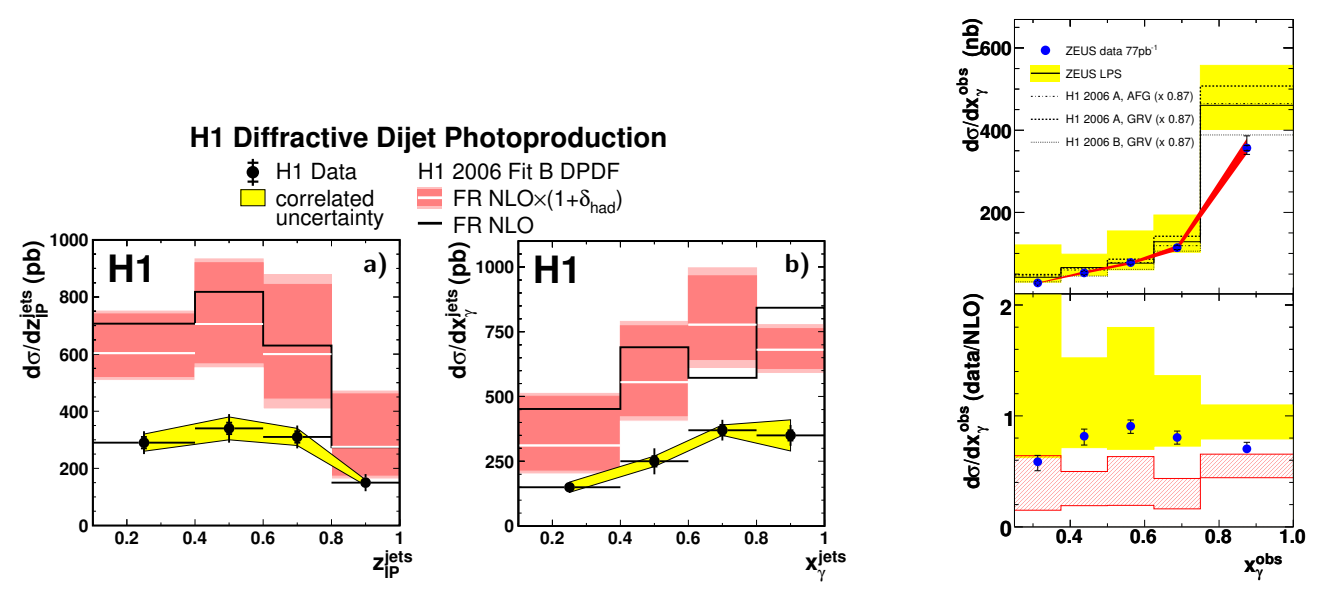

Figure 5: Left: Cross sections $d \sigma / d z_{I P}$ (a) and $d \sigma / d x_{\gamma}^{o b s}$ (b) of diffractive dijets in photoproduction with yellow band of correlated systematic uncertainties. The H1 data are compared to NLO QCD prediction based on H1 2006 dPDFs fit B with a band indicating the scale uncertainty, plots from [4]. Right: ZEUS results on the cross section $d \sigma / d x_{\gamma}^{\text {obs }}$ compared to NLO QCD predictions based on different dPDF inputs, plot from [5].

QCD factorization, broken at Tevatron, can be tested within HERA investigating diffractive dijet photoproduction $\left(Q^{2} \sim 0\right)$, where the hard scale is provided by the $E_{T}$ of the jets. At LO QCD there are two processes contributing to dijet photoproduction: direct photon reactions proceeding via a point-like photon (photon-gluon fusion) and resolved processes where the photon fluctuates into partons. Further interactions from the partons of the photon and of the proton may fill the rapidity gap via rescattering effects discussed also in chapter 4. In principle, factorization is not expected to hold in photoproduction events. However, the suppression of the diffractive cross section could be different for direct and resolved processes. ZEUS [5] and H1 [4] presented precise HERA I data on diffractive dijet photoproduction, see Figs. 5 left and right, using an integrated luminosity of $77 \mathrm{pb}^{-1}$ and 18 $\mathrm{pb}^{-1}$, respectively. $\mathrm{H} 1$ observed a suppression factor of 2 both needed for the direct and the resolved contributions w.r.t. a NLO QCD calculation. ZEUS reported that no suppression is needed to describe the data over the full $x_{\gamma}^{o b s}$ range and the data are well described by a NLO QCD prediction using the H1 2006 B dPDFs. However it has to be noted that the selection criteria differ, in particular in the minimum transverse momentum required for the highest $E_{T}$ jet which is $5 \mathrm{GeV}$ for $\mathrm{H} 1$ and $7.5 \mathrm{GeV}$ for ZEUS. In addition, the covered $x_{\mathbb{P}}$ range is somewhat different with $x_{\mathbb{P}}<0.025$ (0.03) for ZEUS (H1). Following discussions at the workshop, several questions were raised: Do events at lower $E_{T}$ require larger suppressions w.r.t. NLO QCD and the different suppression factors are due to the different $E_{T}$ cuts? Are the two different NLO QCD programs used for the predictions in agreement? How is the charm contribution treated in the NLO calculations? These interesting points are under further careful evaluation by the collaborations and theorists. 
Recently published measurements of diffractive open charm production in DIS and in photoproduction were reported by H1 [6] and by ZEUS [7] using integrated luminosities of about 48 $\mathrm{pb}^{-1}$ and $79 \mathrm{pb}^{-1}$, respectively. Here, the diffractively produced system $X$ should contain at least one charmed hadron which is usually tagged by the reconstruction of a $D^{* \pm}(2010)$ meson. With this method ZEUS determined the ratio of diffractive to inclusive charm cross section to $5.7_{-0.7}^{+0.9} \%$ for $x_{\mathbb{P}}<0.035$ in very good agreement with NLO QCD calculations [7]. Using a second analysis technique based
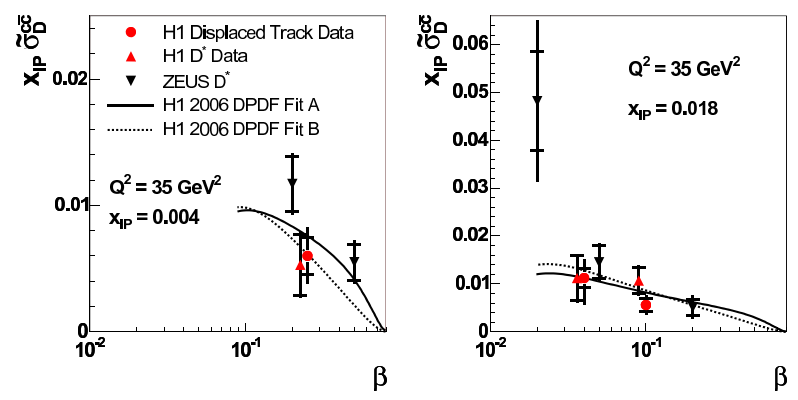

Figure 6: The reduced cross section $x_{\mathbb{P}} \tilde{\sigma}^{c \bar{c}}$, integrated over $|t|<1 \mathrm{GeV}^{2}$, as a function of $\beta$ for two $x_{\mathbb{P}}$ values. The ZEUS and H1 data are compared to recent NLO QCD calculations using H1 2006 dPDFs A and B; plot from [6].

on the displacement of tracks from the primary vertex, H1 measured the diffractive cross section of a charm enriched sample in photoproduction. The measurements are found to be in good agreement between the two methods and with ZEUS results and are well described by NLO QCD prediction, see Fig. 6 confirming the validity of QCD factorization for open charm production in diffractive DIS and photoproduction.

At HERA II, H1 collected with a newly installed Very Forward Proton Spectrometer (VFPS) [8] an integrated luminosity of around $140 \mathrm{pb}^{-1}$ since 2005. The fiber detectors in the Roman pots, located at 218 and $222 \mathrm{~m}$ downstream from the H1 interaction point, tag and measure diffractively scattered protons with a high acceptance in the range $0.01<$ $x_{\mathbb{P}}<0.025$. It was reported that the VFPS acceptance is well suited for dijet studies both in DIS and photoproduction regimes and more precise data in extended kinematic ranges are anticipated to be confronted with theory.

\section{Exclusive diffraction}

The study of hard exclusive reactions, $e p \rightarrow e X p$, where the hadronic system $X$ is a vector meson (VM) like $\rho, \omega, J / \Psi, \ldots$ or a photon (DVCS), provides a very interesting laboratory to test the mechanism of diffraction in dependence of various scales. At HERA, the large accessible range in terms of vector meson masses, of $Q^{2}$ and of $t$ allows the investigation of the transition from the soft, non-perturbative regime described within the Regge phenomenology to the hard regime described by perturbative QCD.

ZEUS presented [9] final results on exclusive $\rho^{0}$ meson production using an integrated luminosity of $120 \mathrm{pb}^{-1}$ in the kinematic range of $2<Q^{2}<160 \mathrm{GeV}^{2}$ and $32<W<180$ $\mathrm{GeV}$. The $\gamma^{*} p \rightarrow \rho^{0} p$ cross section falls steeply with increasing $Q^{2}$ but can not be described by a simple propagator term like $\sigma \propto\left(Q^{2}+M^{2}\right)^{-n}$, where $M$ is the vector meson mass. The cross section is rising with increasing $\gamma^{*} p$ c.m. energy, $\sigma \propto W^{\delta}$. The $\delta$ parameter increases with the scale $Q^{2}+M^{2}$ as expected for a transition from a soft to a hard regime. The values of $\delta$ obtained for cross sections of exclusive $\rho, \phi, J / \Psi$ as a function of $Q^{2}+M^{2}$ show an universal behaviour, see Fig. 7 left. The $t$ dependence of the cross section can be parametrized via a fit $d \sigma / d|t| \propto e^{-b|t|}$. The exponential slope of the $t$ distribution decreases with increasing $Q^{2}+M^{2}$ and levels off with a value of $b \sim 5 \mathrm{GeV}^{-2}$ at about $40 \mathrm{GeV}^{2}$. A 
compilation of the $Q^{2}+M^{2}$ dependence of $b$ parameters shows the same trend for HERA VM and DVCS measurements, see Fig. 7 right. The helicity analysis of the decay-matrix elements of the $\rho^{0}$ indicates s-channel helicity breaking. was used to extract the ratio of longitudinal to transverse $\gamma^{*} p$ cross section as a function of $Q^{2}$ and $W$. The ratio of the cross sections induced by longitudinally and transversely polarized virtual photons is found to increase as a function of $Q^{2}$ but is independent of $W$. This is a somehow surprising observation since it suggests a similar transverse size of the $q \bar{q}$ configurations in the wave functions of $\gamma_{L}^{*}$ and $\gamma_{T}^{*}$. Finally, an effective Pomeron trajectory was extracted with a larger intercept but smaller slope than those found in soft hadron-hadron interactions. The presented extensive studies of the properties of the $\rho^{0}$ meson are qualitatively in agreement with expectations from pQCD. However, none of
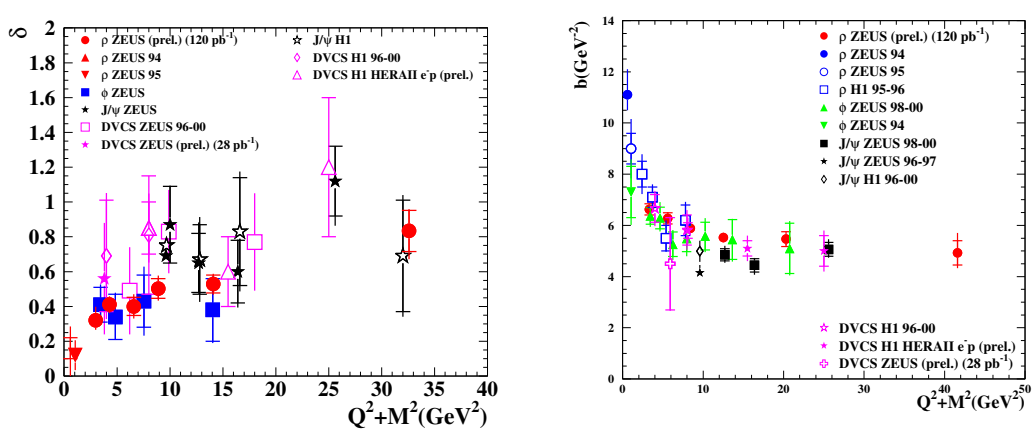

Figure 7: Dependence on the $Q^{2}+M^{2}$ scale of the parameter $\delta$ (left) and $b$ (right) for HERA VM and DVCS data; plot from [9]. the models considered could describe all the observed features of the excellent precision data.

Deeply Virtual Compton Scattering (DVCS), $\gamma^{*} p \rightarrow \gamma p$, consists of the hard diffractive scattering of a virtual photon off a proton. The interest of the DVCS process resides in its clear experimental signature and in the particular insight it gives to the applicability of pQCD in the field of diffractive interactions and to the nucleon partonic structure as described in terms of GPDs.

A new DVCS measurement was presented [10] based on data taken by the H1 detector during the HERA II period using $e^{+}$and $e^{-}$lepton beam data with an integrated luminosity of $145 \mathrm{pb}^{-1}$ each. At the present small values of $|t|$ the reaction $e p \rightarrow e \gamma p$ used to extract
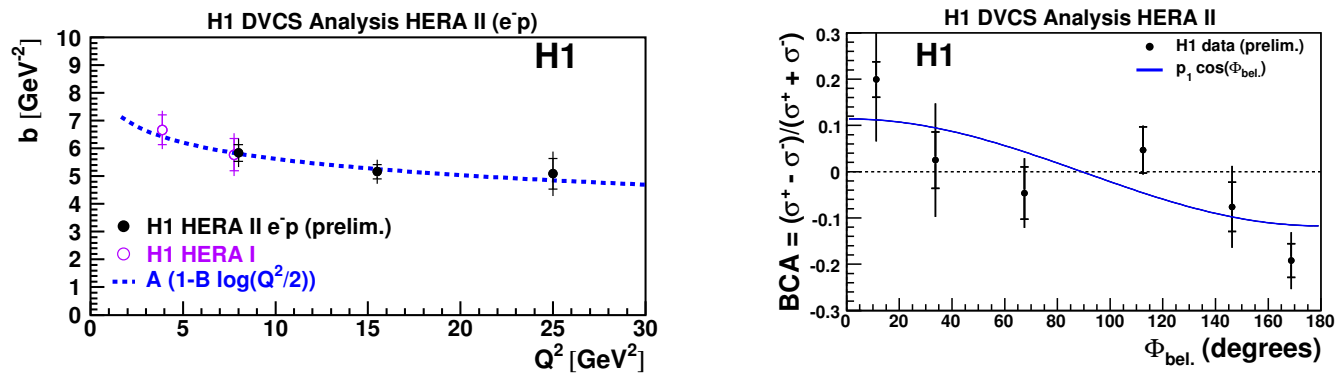

Figure 8: Left: The $t$ slope parameter $b$ as a function of $Q^{2}$. Right: Beam charge asymmetry as a function of azimuthal angle $\phi$; plots from [10]. 
the DVCS signal is dominated by the purely electromagnetic Bethe-Heitler (BH) process whose cross section, depending only on QED calculations and proton elastic form factors, is precisely known and therefore can be subtracted. To enhance the ratio of selected DVCS events to $\mathrm{BH}$ events the outgoing photon is selected in the forward, or outgoing proton, region with transverse momentum larger than $2 \mathrm{GeV}$. The $\gamma^{*} p$ cross sections as a function of $Q^{2}$ and $W$ are well described by QCD based calculations, in particular showing a typical $Q^{2}$ evolution of GPDs. The $t$ dependence is measured and fitting an exponential dependence, $e^{-b|t|}$, the parameter $b$ is found to depend on $Q^{2}$, see Fig. 8 left, and not on $W$. For the first time, a beam charge asymmetry is observed at an $e p$ collider, as shown in Fig. 8 right, combining the $e^{+} p$ and $e^{-} p$ data samples. A significant non zero value is measured, for $|t|>0.05 \mathrm{GeV}^{2}$, related to the interference of the DVCS and Bethe-Heitler processes.

At RHIC, the Phenix experiment uses hadronic and leptonic decays of $\Phi$ mesons as a probe sensitive to media effects induced by an extremely hot and dense matter produced in $\mathrm{Au}+\mathrm{Au}$ collisions at $\sqrt{s_{N N}}=200 \mathrm{GeV}$ [11]. The integrated yields of the $\Phi$ meson productions by $e^{+} e^{-}$and $K^{+} K^{-}$decays were found to be consistent with each other and also independent of the centrality of the collision and so far no media modification could be detected within the present uncertainties. Phenix reported [12] also results on multiparticle decay of $K_{S}^{0}, \eta$, and $\omega$ mesons at high $p_{T}$ in $\mathrm{p}+\mathrm{p}, \mathrm{p}+\mathrm{d}$ and $\mathrm{Au}+\mathrm{Au}$ collisions. No suppression of the meson yields were observed in $\mathrm{p}+\mathrm{d}$ collisions but a strong suppression of $\eta$ and $\omega$ production in $0-20 \%$ most central $\mathrm{Au}+\mathrm{Au}$ events at the same energy.

\section{Leading baryon production at HERA}

The production of leading baryons belongs to one of the most interesting semi-inclusive reactions which may be explored with an electromagnetic probe at the HERA collider. While hard interactions are well understood in the QCD improved parton model, predictions for semi-inclusive processes are not so reliable yet. The applicability of pQCD, if at all, is restricted to the range of events with high $p_{T}$. The production mechanisms at low $p_{T}$ is reasonably described by so-called exchange models: in the $\gamma^{*} p$ reaction the incoming photon is considered as a $q \bar{q}$ state which scatters on a virtual particle emitted from the proton. The outgoing, leading baryon carries a significant fraction of the incoming protons energy, $x_{L}$. From this picture it becomes obvious that in such a process long range properties of the baryon could be analyzed: at which length breaks the proton into two colorless objects, a meson and a nucleon which could be a pion and a neutron in the simplest picture for leading neutron production? For nuclear physics this question is interesting for a further understanding of the role of meson exchange forces in nucleon-nucleon interactions. Regarding high energy physics the factorization hypothesis could be tested: can this process be separated into the interaction and an universal target fragmentation, i.e. is it independent of the probe which initiates the fragmentation? There are predictions that this simple factorization picture is only valid for a well separated nucleon-meson system in the target proton at peripheral collisions where the nucleon acts as a spectator. In more central collisions, larger projectiles like a proton or a real photon (a large sized $q \bar{q}$ pair) can destroy the nucleon through rescattering effects and break the factorization.

At HERA around $10 \%$ of the inclusive DIS events contain a leading baryon. Now the ZEUS collaboration presented its final, high precision HERA I results on the production of energetic neutrons in ep collisions using a lead-scintillator calorimeter and a scintillator hodoscope as a forward neutron detector [13]. The neutron energy and $p_{T}^{2}$ 
distributions were measured in a broad kinematic range, $0.2<x_{L}<1$ and $p_{T}^{2}<$ $0.476 x_{L}^{2} \mathrm{GeV}^{2}$, in a $40 \mathrm{pb}^{-1}$ sample of inclusive DIS data $\left(\sigma_{\text {inc }}\right)$ and a $6 \mathrm{pb}^{-1}$ sample of photoproduction data. The normalized neutron production rate rises due to the increase in $p_{T}^{2}$ space from lowest $x_{L}$ towards its maximum at around $x_{L} \sim 0.7$, and falls to zero at $x_{L}=1$, but the neutron yield in photoproduction is suppressed relative to DIS for the lower neutron energies, see Fig. 9 left. Screening effects lower the neutron yield mainly at lower neutron energies (lower $x_{L}$ ) depending on the transverse size of the $q \bar{q}$ pair: absorptive corrections are expected to be smaller for highly virtual photons (larger $Q^{2}$ ) due to less rescattering of small
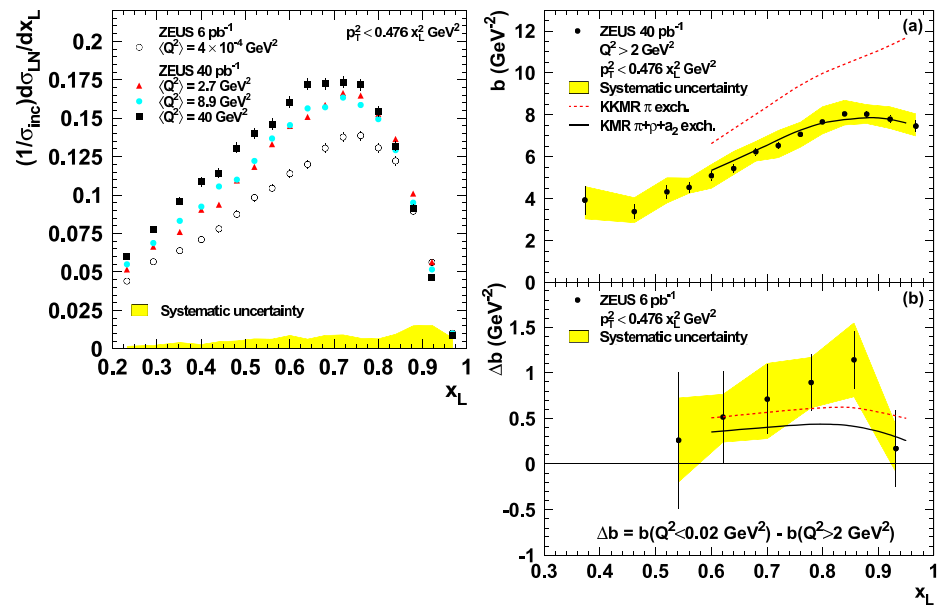

Figure 9: Left: Normalized leading neutron production rate versus $x_{L}$ for three DIS samples and for photoproduction. Systematics common to all samples is shown as a shaded band. Right: (a) Slopes $b$ from an exponential fit to the $p_{T}^{2}$ distribution for DIS and (b) difference of photoproduction and DIS $b$ slopes. The shaded band shows the systematic uncertainty. The dashed curves come from a prediction based on pion exchange with enhanced neutron absorption and migration, the solid curves include also $\rho$ and $a_{2}$ exchanges in the calculation; plot from [13]. size color dipoles on the target fragment neutron. The observed $Q^{2}$ dependence of the neutron yields violates the factorization hypothesis. The $p_{T}^{2}$ distributions are steeper for events in photoproduction, i.e. there are fewer neutrons at higher $p_{T}$. The $\left(x_{L}, p_{T}^{2}\right)$ distribution of the normalized neutron yields can be fully parametrized by an exponential fit with a slope parameter $b\left(x_{L}\right)$ and an intercept $a\left(x_{L}\right)$. The $b$ slopes for photoproduction are larger for the range $0.6<x_{L}<0.9$, see Fig. 9b, indicating the breakdown of vertex factorization. As shown in Fig. 9a, the precision of the data allows to distinguish between the various models: pure one-pion exchange with neutron absorption predicts too large $b$ values, but the addition of subleading $\left(\rho, a_{2}\right)$ Reggeons gives a fair description of both parameters. The model also accounts for migration of neutrons in $\left(x_{L}, p_{T}^{2}\right)$ after rescattering and estimates the loss of neutrons through absorption to about $50 \%$.

None of the Monte Carlo models commonly used for simulating DIS and photoproduction events describe the leading neutron data. Adding to the implemented standard proton fragmentation also diffraction and pion exchanges gives a good description of the shapes of the slopes, although with too large absolute values.

The present data can be used to further constrain exchange models and thus to improve the calculations of diffractive productions at the LHC. 


\section{Diffraction in hadronic collisions}

New diffraction results obtained in proton-antiproton collisions at the Fermilab Tevatron collider at c.m.s. energy of $\sqrt{s}=1.96 \mathrm{TeV}$ (Run II) confirm and extend important observations made at lower c.m.s. energies of $0.63 \mathrm{TeV}$ and $1.8 \mathrm{TeV}$ (Run I). For the diffractive program at Run II, the CDF collaboration instrumented the detector with special forward components [14] : a Roman Pot spectrometer (RPS) to detect leading antiprotons (improved acceptance for small $|t|$ in comparison to Run Ic), Mini Plug calorimeters to cover the region of about $3.5<|\eta|<5.2$, and beam shower counters positioned along the $p$ and $\bar{p}$ beam directions to tag gaps within $5.5<|\eta|<7.5$.

In hard single diffraction, CDF obtained preliminary Run II results for the Bjorken- $x$ and $Q^{2}$ dependence of the diffractive structure function from the ratio of two dijet samples: single diffractive (SD) dijets triggered by an intact antiproton in the RPS and non-diffractive (ND) dijet events. This ratio is in LO QCD approximately equivalent to the ratio of the corresponding structure functions. The diffractive structure function versus Bjorken- $x$, the momentum fraction of a parton in the antiproton, confirms the factorization breakdown by about factor of 10. In Run II, higher $E_{T}^{\text {jet }}$ energies up to $100 \mathrm{GeV}$ could be recoreded thus allowing decent tests of the $Q^{2}$ dependence, where $Q^{2}$ is here given by the value of $<E_{T}^{*}>^{2}$. In the range of $100<Q^{2}<10000 \mathrm{GeV}^{2}$, no significant dependence on the $Q^{2}$ scale could be observed for the $\mathrm{SD} / \mathrm{ND}$ dijet ratio, although in this range the inclusive $E_{T}$ distribution falls by a factor of 10000 . This result suggests that the $Q^{2}$ evolution of diffractive and non-diffractive interactions is similar. The $t$ dependence of the diffractive cross section was explored by a double exponential fit, no diffraction dips were observed for the range $|t|<1$ $\mathrm{GeV}^{2}$ and no strong $Q^{2}$ dependence of the slope parameter $\left.b\left(Q^{2}\right)\right|_{t=0}$.

With the advent of the luminosity upgrade of Tevatron, the diffraction program of CDF could be extended to the study of central exclusive production in $p \bar{p}$ collisions. This process is not only of interest for testing QCD inspired models of hard diffraction but also due to its discovery potential. Exclusive Higgs production, $p+p \rightarrow p+H+p$, is expected to provide clean events with suppressed QCD background and a precise measurement of the Higgs mass based on the momentum measurements of the two outgoing protons. However, those advantages are hampered by the low production rates predicted. The rate calculations suffer from considerable model dependencies due to the unknown non-perturbative suppression

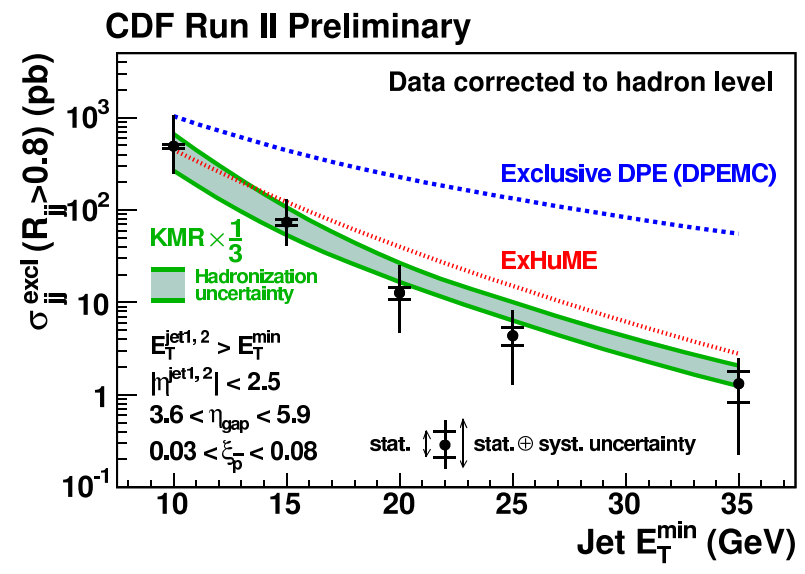

Figure 10: Exclusive dijet cross section measured from data as a function of minimum second jet $E_{T}$. The dotted (dashed) lines show the ExHuMe (exclusive DPE in DPEMC) Monte Carlo predictions. factors. While exclusive Higgs boson production is not in the reach at Tevatron energies, exclusive dijet production, $\bar{p}+p \rightarrow$ $\bar{p}+J e t+J e t+p$, could be considered as a rather high rate process which proceeds through 
the same mechanism and could allow the determination of the suppression factor experimentally.

CDF presented first results on central exclusive dijet cross sections, see Fig. 10, produced in Double Pomeron Exchange (DPE) based on a data sample of about $310 \mathrm{pb}^{-1}$ collected with a dedicated diffractive trigger in Run II [14]. Exclusive dijet searches use a measurement of the dijet mass fraction, $R_{j j}$, defined as the ratio of the mass of the two leading jets in an event, $M_{j j}$, to the total mass, $M_{X}$. Exclusively produced dijet events could be observed as a significant excess of data at large $R_{j j}$ by comparing the Rjj distribution shapes between the data and inclusive DPE dijet Monte Carlo predictions. Also shown in Fig. 10 is a LO calculation for exclusive Higgs boson production at the LHC downscaled by factor of three. The agreement between calculation and data is encouraging keeping in mind experimental and theoretical uncertainties.

Like exclusive dijet production, exclusive $\gamma \gamma$ production could be used for calibrating diffractive Higgs boson production models. Based on three events, CDF reported for the first time a $95 \%$ C.L. upper limit of $410 \mathrm{fb}$ on the cross section, $\bar{p}+p \rightarrow \bar{p}+\gamma+\gamma+p$, [14]. The robustness of the exclusive event selection was checked by a measurement of the purely QED process, $\bar{p}+p \rightarrow \bar{p}+e^{+} e^{-}+p$, which was found to be in good agreement with the expectation.

At the workshop comprehensive reports on the forward and very forward physics projects and the status of the relevant detectors for the LHC experiments CMS [15] and ATLAS [16] were presented. Within both collaborations the installation of Roman Pot stations at $420 \mathrm{~m}$, as investigated by the FP420 R\&D Collaboration [17], is under discussion. New studies were presented [18] showing that additional roman pot stations at $220 \mathrm{~m}$ nicely complement the FP420 project. Combined $220 \mathrm{~m} / 420 \mathrm{~m}$ detectors could achieve acceptances of at least $30 \%$ for a large missing mass range of 100 until $800 \mathrm{GeV}$ which is important for a discovery of the Higgs boson in exclusive reactions. Lively discussed were questions regarding the trigger, the technology for the pots, the position and the timing detectors, e.g. requirements of 5 to 10 ps resolutions are discussed for a fast timing needed to reject background from pile-up events.

\section{New theoretical developments}

The standard QCD description of the inclusive hard diffraction is based on the Operator Product Expansion and DGLAP evolution of collinear diffractive parton densities. Limits of validity of this framework were investigated [19] when the proton mass $M_{P}$ and momentum transfer $t$ cannot be neglected. The twist 2 contributions to the structure functions were studied. In the limit of $M_{P} \rightarrow 0$ and $t \rightarrow 0$ the expected results were found: there exist two structure functions in the unpolarized case connected at leading order by a modified CallanGross relation. In the polarized case, four structure functions appear, that are connected by an all order Wilczek-Wandzura relation. All these structure functions may be expressed in terms of diffractive parton densities. This picture changes at low $Q^{2}$ and the large $\beta$ where it is necessary to extend the analysis beyond the $M_{P} \rightarrow 0$ and $t \rightarrow 0$ limit. Then, the number of structure functions grows to four (eight) in the unpolarized (polarized) scattering case. Moreover, the connection of those structure functions to the diffractive parton densities is lost.

The region of low $Q^{2}$ and large $\beta$ is interesting also because large higher twist corrections to the dDIS cross section should appear there. One of the reasons is, that the twist 2 contri- 
butions vanish as $\beta \rightarrow 1$, while it is not the case for twist 4 . In particular, the contribution to the diffractive cross section coming from longitudinally polarized virtual photons is known to be important at large $\beta$. This contribution may be evaluated perturbatively within the $k_{T}$-factorization framework. Thus, it was proposed to fit the dDIS data within a modified DGLAP approach, in which the twist 4 contribution is subtracted from the data [20, 21]. The modified fits give values of $\chi^{2}$ only slightly worse than the conventional twist 2 DGLAP fit. The diffractive quark densities emerging from the modified fit are slightly lower w.r.t. the twist 2 case while the gluon density is enhanced at large $\beta$, and the effect is particularly pronounced at lower $Q^{2}$. It turns out, that inclusion of the twist 4 contribution increases dramatically the predictions for $F_{L}^{D}$ at $\beta>0.5$ and typical values of $Q^{2}$. Thus, it should be very interesting to measure $F_{L}^{D}$ at large $\beta$.

The diffractive scattering may be viewed as a manifestation of unitarity corrections to a single (cut) Pomeron exchange contribution to the total cross-section. In addition, in contrast to the inclusive DIS the diffractive deep inelastic scattering amplitude is dominated by hadronic fluctuation of large sizes, even though the photon virtuality is large. Thus, the dDIS is intimately connected to the effects of parton saturation and it probes unitarity corrections to the QCD evolution of parton densities. This connection was exploited in the very successful saturation model, proposed by Golec-Biernat and Wüsthoff (GBW). Since then the simple saturation model was extended to incorporate effects of QCD evolution and of the proton shape; it was also used to describe a broader spectrum of observables. In particular, it was shown that saturation models provide a rather good description of exclusive diffractive processes: exclusive vector meson photo- and electroproduction and DVCS at small $x$, including the $t$-dependence $[22,23]$.

This complementary approach to the hard diffraction is based on the $k_{T}$-factorization. The theoretical framework is rooted in the Balitsky-Fadin-Kuraev-Lipatov formalism for rapidity evolution of high energy scattering amplitudes into which some unitarity corrections are incorporated (e.g. multiple scattering or gluon recombination). In this approach the issues of unitarity corrections and diffractive scattering at high energies may be conveniently addressed in the transverse position space. Along this line an interesting generalization of the GBW approach to the hard diffraction was developed [24]. The hadronic multiparton state that scatters diffractively was constructed using Mueller's color dipole model of BFKL dynamics. In the large $N_{c}$ limit the hadronic state may be decomposed into color dipoles. The coherent ensemble of QCD dipoles scatters multiply and the scattering amplitude obeys unitarity constraints. Thus, the projectile evolution and the scattering process are factorized at the rapidity corresponding to the rapidity gap size. The multidipole scattering amplitudes should follow from small- $x$ evolution equations obtained within the so called Color Glass Condensate (CGC) framework. The main merit of this approach is a possibility to incorporate an arbitrary number of (soft) gluons in the scattering state. Unfortunately, exact solutions to the CGC evolution equations at full accuracy (that is including the Pomeron loops) are not yet known. Thus these amplitudes still have to be modeled. The problem of diffractive scattering beyond the large $N_{c}$ limit was addressed in terms of the JIMWLK evolution equation [25]. The structure of this equation is similar to the Balitsky-Kovchegov (BK) equation, that is the Pomeron loops are not well represented. In contrast to the BK equation, however, the JIMWLK evolution may generate multi-gap diffractive final states. It turns out that the difference originates from subleading effects in the $1 / N_{c}$ expansion which lead to color reconnection and formation of additional rapidity gaps. 
Recently, new extensions of saturation models to exclusive processes were put forward. A new model of the color dipole scattering amplitude was constructed, based on an analysis of the non-forward BK equation in full momentum space [23]. An interesting feature of this model is the presence of a saturation scale that depends on the momentum transfer and not on the transverse position. The model provides a very good description of the light vector meson data at small $x$. Another approach is based on the Glauber-Mueller formula assuming multiple independent scatterings [22]. The model is constructed using spatial coordinates: the dipole size $r$ and transverse position $b$. The LO DGLAP effects of the gluon evolution are incorporated and the $b$-dependence of the single scattering amplitude $T \Omega(r, b)$ is assumed to take a factorized form: $\Omega(r, b) \sim x g\left(x, \mu_{0}^{2}+C / r^{2}\right) S(b)$, where $\mu_{0}$ provides a freeze-down scale for the DGLAP evolution, and $S(b)$ describes the proton shape. Within this model a reasonably good unified description of the inclusive observables (including shadowing effects in DIS on nuclei) and most of exclusive diffractive observables was obtained. It was found that a Gaussian proton shape $S(b) \sim \exp \left(-b^{2} / R^{2}\right)$ is strongly preferred to a box-like shape $S(b) \sim \Theta(R-b)$. The radius of the gluon distribution in the proton was determined to be $R=0.69 \mathrm{fm}$, a value smaller than the electromagnetic Hofstadter proton radius $R_{H}=0.870 \pm 0.008 \mathrm{fm}$. Saturation effects were found to be moderate, at HERA: for $x>10^{-4}$ one obtains $Q_{s}^{2}(b)<1 \mathrm{GeV}^{2}$ for $b=0 \mathrm{fm}$ and $Q_{s}^{2}(b)<0.4 \mathrm{GeV}^{2}$ for $b=0.4 \mathrm{fm}$. Another version of the saturation model was successfully applied also for heavy quark production at HERA [26]. The flavored structure functions $F_{2}^{c}$ and $F_{2}^{b}$ were described together with $F_{2}$ and $F_{2}^{D(3)}$ within a saturation model with DGLAP evolution, but without the impact parameter dependence. The best fit to $F_{2}$ with $\chi^{2} /$ d.o.f. $=1.16$ is significantly improved with respect to the GBW fit without QCD evolution. The best fit required, however, a surprisingly large freeze-down scale $\mu_{0}^{2}=1.6 \mathrm{GeV}^{2}$. It is interesting to note that the fits based on the saturation model with the QCD evolution and heavy flavors imply an almost flat $x$ dependence of the gluon density $x g\left(x, \mu_{0}^{2}\right)$ at the low scale.

An important feature of the GBW saturation model is the geometric scaling property: the total $\gamma^{*}\left(Q^{2}\right) p$ cross section depends only on one variable $\xi=Q^{2} / Q_{s}^{2}(x)$ where $\log Q_{s}(x) \sim$ $\log (1 / x)$. The geometric scaling was actually found in the HERA data and, interestingly enough, it is also a universal property of the solutions of the Balitsky-Kovchegov equation at large rapidities. In fact, the geometric scaling emerges in a wider class of non-linear evolution equations incorporating phenomena of growth and saturation. Inclusion of the QCD running coupling into the BK equation leads to a modified form of geometric scaling, $\log Q_{s}(x) \sim \sqrt{\log (1 / x)}$. It was verified [27] that: (i) the asymptotic solution to the BK equation with the running coupling and non-leading BFKL kernel, is universal and does not depend on details of the treatment of the non-leading QCD effects beyond the running of $\alpha_{s}$, and (ii) the HERA $F_{2}$ data agree well with the modified geometric scaling. It should be remembered, however, that the HERA data probe the gluon density evolution in a rather limited range of rapidities. In this range, some violations of geometric scaling should be still visible due to pre-asymptotic effects. It was also pointed out that some models used in the literature, of the dipole cross section that incorporate geometric scaling violations differ significantly from solutions to the BK equation [28].

In most of the applications of the color dipole model developed so far one assumes that the dipole cross section $\sigma_{d}(x, r)$ depends only on the $x$ variable and the dipole size $r$. This assumption is not obvious at all - it was argued $[29,30]$ that an alternative choice of $\sigma_{d}\left(W^{2}, r\right)$ which depends on $r$ and on the photon-proton collision energy, $W$, could be more 
natural. The choice of $W$ as the relevant variable imposes strong constraints on the ratio of DIS cross sections with longitudinal and transverse photons $R=\sigma_{L} / \sigma_{T}<0.37$ [30]. It was noticed [29], however, that this bound is completely removed if one improves the treatment of the photon wave function by limiting the allowed invariant masses of the $q \bar{q}$ fluctuations of the virtual photon.

Exclusive vector meson production data provide a lot of insight into proton structure and QCD dynamics. For instance, $J / \psi$ photo- and electroproduction is a direct and sensitive probe of the gluon density. It was proposed to use the data for the exclusive photoproduction of $J / \psi$ at HERA to constrain the gluon density beyond the leading logarithmic approximation [31]. The dependence of the hard matrix element to the gluon momentum was taken into account within the $k_{T}$ factorization framework, and the unintegrated gluon density was obtained from the collinear gluon density using the Kimber-Martin-Ryskin prescription. This construction aims at accounting important non-leading corrections to the collinear LO formula, that are predominantly of kinematic origin. The gluon densities that emerge from the fit are flatter functions of gluon $x$ than the LO MRST and CTEQ parameterizations, getting close to the NLO and NNLO gluon densities. A good description of the light vector meson electroproduction data that range from the fixed target kinematics down to small $x$ was obtained [32] within a collinear factorization approach with GPDs at the LL accuracy. It was necessary, however, to use a model of the meson wave function which incorporates the quark transverse momentum — with the leading twist distribution amplitude the data are badly underestimated. This result is interpreted as a manifestation of large power corrections to the exclusive $\rho^{0}$ production amplitude.

Meson impact factors at NLO accuracy that were calculated in recent years are now used in estimates of the NLO effects in meson production amplitudes. A detailed analysis of the NLO effects in the longitudinally polarized $\rho^{0}$ meson photoproduction was performed [33]. It follows from this analysis that the perturbative expansion is rather stable at large and moderate Bjorken $x$, corresponding to the kinematics of fixed target experiments. At small $x$, however, the NLO corrections are enhanced by $\log (1 / x)$, and they are comparable to the leading amplitudes, even at pretty large values of the photon virtuality $Q^{2}$. For instance, at $x=2 \cdot 10^{-3}\left(x=2 \cdot 10^{-4}\right)$ the NLO correction is still dangerously large at $Q^{2}=16 \mathrm{GeV}^{2}$ (at $Q^{2}=49 \mathrm{GeV}^{2}$ ). This questions both the reliability of the LL calculations and the stability of the fixed order perturbative calculations in the small $x$ domain and at moderate $Q^{2}$.

The generalized parton densities of the nucleon emerge partially from non-perturbative QCD dynamics. Thus, within perturbative QCD it necessary to determine them from fits to experimental data. An interesting attempt was made [34] to improve this situation and to use QCD simulations on the lattice to constrain the nucleon GPDs the flavor non-singlet sector. As a result new parameterizations of those GPDs were obtained in which the model dependence is expected to be reduced w.r.t. the conventional parameterizations.

An excellent probe of the BFKL effects is given by an exclusive production of two vector mesons in two virtual photon collisions. Such measurements should be possible at future $e^{+} e^{-}$colliders. The process of $\gamma^{*} \gamma^{*} \rightarrow \rho_{L}^{0} \rho_{L}^{0}$ in the high energy limit was studied [35] in the framework of the NLL BFKL, including the impact factors at the NLL accuracy. The sensitivity of the amplitude to the scheme choice, the factorization scale and the energy scale was systematically analyzed. The NLL predictions are found to be stable against variations of the scheme and of the scales. A rather surprising result was obtained that the NLL BFKL cross section at moderate energies is much lower than its lower order approximation of a two-gluon exchange. A complementary study of the double $\rho^{0}$ meson production in two 
photon collisions was carried out for moderate collision energies, where a quark exchange dominates over the BFKL amplitude. An interesting pattern of QCD factorization(s) was found there [36]. Namely, the QCD factorization of the amplitude for $\gamma^{*}\left(Q_{1}^{2}\right) \gamma^{*}\left(Q_{2}^{2}\right) \rightarrow$ $\rho_{L}^{0} \rho_{L}^{0}$ at a moderate c.m.s. energy squared $W^{2}$ may be performed using usual BrodskyLepage distribution amplitudes. For $\gamma_{T}^{*}\left(Q_{1}^{2}\right) \gamma_{T}^{*}\left(Q_{2}^{2}\right) \rightarrow \rho_{L}^{0} \rho_{L}^{0}$ at $W^{2} \ll Q_{i}^{2}$ the factorization into the hard matrix element and a generalized distribution amplitude works. Finally, when the photon virtualities are strongly ordered, $Q_{1}^{2} \gg Q_{2}^{2}$ a hard factorization is possible with a suitable transition distribution amplitude. Thus, in the exclusive double $\rho^{0}$ photoproduction (with virtual photons) one may probe in detail various features of QCD dynamics and the meson structure.

Exclusive $C$-even meson photoproduction is driven by the $C$-odd gluonic exchange i.e. the QCD Odderon instead of the Pomeron, Therefore, an exclusive $\pi^{0}$ photoproduction off the proton was expected to be a sensitive probe of the Odderon. In the measurement performed at HERA, however, no signal was found: the upper limit for the exclusive cross section was found to be $49 \mathrm{nb}$, much below theory expectations of about $300 \mathrm{nb}$. It was suggested that this puzzling discrepancy may be removed when the theoretical estimates properly take into account the chiral symmetry of QCD [37]. In the preceding theoretical estimate of the cross section the constraints imposed by the chiral symmetry were neglected and the cross section was overestimated.

Shortly before the LHC is going to start, hard exclusive diffractive processes in $p p$ collisions receive a lot of attention. The highlight here are possible studies of the Higgs boson in the exclusive channel, using forward proton detectors e.g. in the FP420. The main merit of the exclusive Higgs boson production process is a strong background suppression and potential precision of the measurements of the Higgs boson properties, including the quantum numbers, the mass and the width. On the other hand, the price to be paid is a reduction of the signal by four orders of magnitude with respect to the inclusive case. Nevertheless, there are chances that the signal is visible, especially if a supersymmetric scenario with large $\tan \beta$ is realized.

From the theory side, the QCD approach elaborated by the Durham group (the KMR model) remains the reference calculational framework. It is assumed that the exclusive diffractive amplitude may be factorized into a hard production amplitude calculable in perturbative QCD, and soft rescattering of spectator partons. The soft part represented by a gap survival factor, $S^{2}$, is estimated from models of the unitary $S$ matrix fitted to data on $p p / p \bar{p}$ scattering. An independent theoretical analysis of this framework foundations confirmed its validity [38]. It was argued that independence of the hard production and the soft rescattering follows from essential difference in space-time scales characteristic for these subprocesses. Also, the obtained value of $\hat{S}^{2}$ agreed well with the value determined by the Durham group. A still open problem remains, however, of the relevance of hard rescattering corrections both of the spectators and within the hard Higgs boson production amplitude. It is therefore encouraging that the data from Tevatron on exclusive diphoton and dijet production are reasonably well described within the KMR model [14]. An analysis using a Monte-Carlo code DPEMC clearly shows that a model of hard exclusive production based on a non-perturbative approach (originally proposed by Białas and Landshoff for diffractive Higgs boson production) is not able to describe the invariant mass dependence of the exclusive dijet production [21], see Fig. 10. Another model for diffractive scattering, the Soft Color Interaction model (SCI), is based on random color reconnections between the hard partons in the final state. The model has been also implemented in the DPEMC. The 
exclusive jet production cross section from SCI was shown to underestimate the Tevatron data.

A key problem in the Higgs boson searches in the exclusive production process at the LHC is a good background control. The $b \bar{b}$ decay channel favored for low Higgs boson masses $M_{H}<130 \mathrm{GeV}$ has an irreducible background from central exclusive production of $b \bar{b}$ dijets. In addition, a reducible background comes from multiple events in a single bunch crossing - the overlap events. Detailed simulations based on Monte-Carlo implementations of diffractive physics, ExHuME, POMWIG and HERWIG+JIMMY, showed that it should be possible to perform a significant $(3-4 \sigma)$ measurement of the exclusive Higgs boson events provided that a supersymmetric scenario with large $\tan \beta$ is realized [17]. A precise time-of-flight analysis is necessary to suppress the background from overlap events. For instance, with suitably tuned cuts on the dijet kinematics and the underlying event, the supersymmetric Higgs boson with $\tan \beta=40$ and $M_{H}=119.5 \mathrm{GeV}$ can be measured in the exclusive channel with the cross-section (after cuts) of about $0.5 \mathrm{fb}$ with a signal to background ratio of about one. The reducible background from overlap events is negligible in a low luminosity mode $\mathcal{L} \simeq 10^{33} \mathrm{~cm}^{-2} \mathrm{~s}^{-1}$ but it is as large as the signal at high luminosities. As a result the significance of the exclusive Higgs boson measurement does not grow with the LHC luminosity beyond $\mathcal{L} \simeq 2 \times 10^{33} \mathrm{~cm}^{-2} \mathrm{~s}^{-1}$.

\section{Acknowledgments}

We would like to thank warmly all session participants and contributors for making the session lively and interesting. We are grateful to the organizers for hospitality and assistance. We thank our co-covenors Rolf Ent and Laurent Favart for organizing the session, and Laurent Favart for his help in preparing the write-up.

\section{References}

[1] Slides: http://indico. cern. ch/contributionDisplay $\cdot$ py?contribId=15\&sessionId=2\&conf $I d=9499$

[2] J. Lukasik, these Proceedings.

[3] B. Löhr, these Proceedings.

[4] M. Mozer, these Proceedings.

[5] Y. Yamazaki, these Proceedings.

[6] P. Thompson, these Proceedings.

[7] I. Melzer-Pellmann, these Proceedings.

[8] L. Favart, these Proceedings.

[9] A. Levy, these Proceedings.

[10] L. Schoeffel, these Proceedings.

[11] S. Huang, these Proceedings.

[12] A. Milov, these Proceedings.

[13] W. Schmidke, these Proceedings.

[14] C. Mesropian, these Proceedings.

[15] K. Borras, these Proceedings.

[16] S. Ask, these Proceedings.

[17] A. Pilkington, these Proceedings.

[18] C. Royon, these Proceedings.

[19] J. Blümlein, B. Geyer and D. Robaschik, these Proceedings; Nucl. Phys. B 755 (2006) 112.

[20] K. Golec-Biernat and A. Łuszczak, arXiv:hep-ph/0704.1608. 
[21] O. Kepka and C. Royon, these Proceedings; C. Royon et. al., arXiv:hep-ph/0609291.

[22] H. Kowalski, these Proceedings; H. Kowalski, L. Motyka and G. Watt, Phys. Rev. D 74 (2006) 074016

[23] R. Peschanski, C. Marquet and G. Soyez, these Proceedings; arXiv:hep-ph/0702171.

[24] C. Marquet, these Proceedings; Y. Hatta et. al., Nucl. Phys. A 773 (2006) 95.

[25] M. Lublinsky, these Proceedings; A. Kovner, M. Lublinsky and H. Weigert, Phys. Rev. D 74 (2006) 114023.

[26] S. Sapeta, these Proceedings; K. Golec-Biernat and S. Sapeta, Phys. Rev. D 74 (2006) 054032.

[27] G. Beuf, R. Peschanski and S. Sapeta, these Proceedings; G. Beuf and R. Peschanski, Phys. Rev. D 75 (2007) 114001.

[28] D. Boer, A. Utermann and E. Wessels, these Proceedings; Phys. Rev. D 75 (2007) 094022.

[29] D. Schildknecht, these Proceedings.

[30] C. Ewerz and O. Nachtmann, Phys. Lett. B 648 (2007) 279.

[31] T. Teubner, these Proceedings.

[32] P. Kroll, these Proceedings; S. V. Goloskokov and P. Kroll, Eur. Phys. J. C 50 (2007) 829.

[33] M. Diehl and W. Kugler, these Proceedings; arXiv:0708.1121 [hep-ph].

[34] S. Liuti, these Proceedings; S. Ahmad et. al., arXiv:0708.0268 [hep-ph].

[35] D. Ivanov, A. Papa, these Proceedings; Eur. Phys. J. C 49 (2007) 947.

[36] M. Segond, these Proceedings; B. Pire et. al., Phys. Lett. B 639 (2006) 642.

[37] C. Ewerz and O. Nachtmann, these Proceedings; Eur. Phys. J. C 49 (2007) 685.

[38] C. Weiss, these Proceedings; L. Frankfurt et. al., Phys. Rev. D 75 (2007) 054009. 